\title{
Forma urbana y Huella Ecológica en el Área Metropolitana de Concepción (Chile)
}

Iván Muñiz. Universitat Autònoma de Barcelona, Barcelona, España.

Carolina Rojas. Universidad de Concepción, Concepción, Chile.

Carles Busuldu. Geintec Asesorías, geografía, información y tecnología, Concepción, Chile.

Alejandro García. Universitat Autònoma de Barcelona, Barcelona, España.

Mariana Filipe. Universitat Autònoma de Barcelona, Barcelona, Espańa.

Marc Quintana. Geintec Asesorías, geografía, información y tecnología, Concepción, Chile.

RESUMEN | Uno de los aspectos más controvertidos del debate sobre sostenibilidad urbana es hasta qué punto el impacto ambiental global de las ciudades está condicionado por su forma y estructura espacial. El Enfoque de Ciudad Compacta sostiene que las zonas más densas y céntricas de las grandes ciudades presentan un menor impacto ambiental per cápita. La densidad poblacional y la distancia al centro de negocios principal ( $\mathrm{CBD}$ ) han sido las variables más utilizadas para capturar la morfología de las ciudades. En este trabajo se ha estimado la Huella Ecológica de los consumos energéticos residenciales y de la movilidad obligada y por motivos de ocio a partir de 475 encuestas llevadas a cabo en el Área Metropolitana de Concepción (Chile). Una vez controlados los posibles problemas de endogeneidad y tenidos en cuenta los aspectos socioeconómicos que pueden incidir en el valor de la huella, la densidad residencial no ejerce una influencia significativa, por lo que las políticas de compacidad podrían resultar poco efectivas. Los resultados obtenidos descartan que dicho resultado se deba a la existencia de comportamientos compensatorios que se traduzcan en una movilidad por ocio anormalmente elevada en los lugares más densos. La renta per cápita es el principal elemento que explica la variabilidad observada en los valores de huella.

PALABRAS CLAVE | indicadores ambientales, demografía, sustentabilidad urbana.

ABSTRACT | One of the most controversial aspects of the debate on urban sustainability is to what extent the overall environmental impact of cities is determined by its shape and spatial structure. Compact City Approach defenders argue that people living in the densest and central areas of large cities have a low environmental impact. Residential density and distance to the Central Business District ( $C B D$ ) are the most common indicators proposed to capture urban spatial structure of cities. In this paper we estimate the ecological footprint of residential energy consumption, commuting and leisure purposes mobility from 475 surveys conducted in Concepción Metropolitan Area (Chile). After controlling for potential endogeneity problems and taken into account the socioeconomic aspects that may affect the value of the Ecological Footprint, residential density does not exercise significant influence, so compactness policies could prove ineffective. The results rule out that this result is due to the existence of compensatory behaviors that result in abnormally high mobility for leisure purposes in denser areas. Income is the main element explaining the observed variability in footprint values.

KEY WORDs | environmental indicators, demography, urban sustainability.

Recibido el 5 de febrero de 2015, aprobado el 3 de septiembre de 2015

E-mail: I. Muniz, ivan.muniz@uab.es |C. Rojas, crojasq@udec.cl | C. Busuldu, c.busuldu@gmail.com | A. García, agarcialdl@gmail.com |

M. Marques, marianafm23@gmail.com | M. Quintana,m.quintana@gmail.com 


\section{Introducción}

Más de la mitad de la población mundial vive en zonas urbanas (UN-Habitat, 2012). Durante las últimas décadas, la expansión física de las ciudades se ha acelerado en prácticamente todas las regiones del planeta (Angel, Sheppard \& Civco, 2005; McGregor, Simon \& Thompson, 2006; United Nations Population Fund [UNFPA], 2007). Las ciudades requieren para su normal funcionamiento de la entrada de alimentos, bienes y energía desde el exterior (Odum, 1971, 1994; Rees, 1992; Vitousek, Ehrlich, Ehrlich \& Matson 1986; Wackernagel \& Yount, 1998); y en tanto lugares de consumo, su contribución a las emisiones de Gases de Efecto Invernadero (GEI) se ha estimado entre el $40 \%$ y el $70 \%$ del total mundial (Satterthwaite, 2008; Walraven, 2009). Diversos trabajos sostienen que la forma y estructura espacial inciden de forma significativa sobre el volumen de emisiones, por lo que el urbanismo y la planificación territorial pueden desempeñar un papel relevante en la lucha contra el cambio climático (Legg, Moore, Kissinger \& Rees, 2013; Muñiz, Calatayud \& Dobaño, 2013; Newman, 2014). Crítico con la creciente dispersión de las ciudades, el llamado Enfoque de Ciudad Compacta concede una importancia crucial a la densidad urbana como mecanismo para frenar su impacto ambiental a escala global (Commission of European Communities [CEC], 1990; Ewing, Bartholomew, Winkelman, Walters \& Chen, 2008; Næss, 2001; Newman \& Kenworthy, 1999; Owens, 1986; Rogers, 2000).

El principal objetivo de esta investigación es estimar el impacto de la forma/ estructura espacial sobre la Huella Ecológica individual de la movilidad y de la energía utilizada en usos residenciales en el Área Metropolitana de Concepción, a través de un modelo de regresión múltiple. Para ello se estima, en primer lugar, la Huella Ecológica correspondiente al volumen de las emisiones individuales a partir de la información que aportan los individuos en cuestionarios pormenorizados expresamente diseńados. La Huella Ecológica es un indicador de in-sostenibilidad que mide la superficie requerida para obtener los recursos y absorber los residuos de una determinada población (Rees \& Wackernagel, 1996). A continuación se estima un modelo de regresión múltiple que presenta las siguientes particularidades:

a. La variable dependiente es la Huella Ecológica de la movilidad y del consumo de energía de las viviendas, incluyendo la movilidad de fin de semana y vacaciones y los consumos energéticos de segundas viviendas. De este modo, podemos detectar la existencia de comportamientos compensatorios (la población que reside en barrios densos presentaría una mayor movilidad durante los fines de semana y periodo vacacional) (Holden, 2007; Hoyer \& Holden, 2003; Muñiz et al., 2013; Næss, 2006);

b. Utiliza dos medidas de forma/estructura espacial: la densidad (más común) y la distancia al centro de negocios principal (CBD) (menos común);

c. Incorpora información individual de naturaleza socioeconómica; y

d. Se controla la endogeneidad asociada a la autoselección, que puede sesgar el valor de los parámetros mediante la inclusión de una variable que captura las 
preferencias de los individuos sobre los diferentes modos de transporte y su importancia a la hora de escoger su localización (Hoden \& Linnerud, 2011; Muñiz et al., 2013; Næss, 2006).

El Enfoque de Ciudad Compacta apuesta por un modelo de ciudad densa y centralizada como estrategia para reducir el volumen de emisiones de Gases de Efecto Invernadero (GEI). En este contexto resulta pertinente analizar el caso de Concepción (Chile), dado que se trata de una ciudad latinoamericana poco densa y descentralizada. ¿Presenta la AMC una elevada huella en movilidad y vivienda debido al hecho de que su forma y estructura espacial sea poco densa y descentralizada? Romero Lankao sostiene que los países latinoamericanos han experimentado un incesante aumento en sus emisiones, por lo que debieran plantear estrategias, también en el campo del urbanismo, que permitiesen reconducir la situación (Romero Lankao, 2007; Romero Lankao, López Villafranco, Rosas Huerta, Gunther \& Correa Armenta, 2005). En claro contraste con las predicciones que se desprenden del Enfoque de Ciudad Compacta, la Huella Ecológica per cápita estimada en esta investigación es baja en relación con los valores obtenidos en otros estudios de naturaleza similar. En cuanto al impacto de la forma/estructura espacial sobre la huella per cápita, la densidad no ejerce un impacto significativo, pero sí lo hace la distancia al centro histórico. De aquí puede inferirse que las políticas basadas en promover tejidos urbanos densos podrían no ser efectivas.

\section{Sostenibilidad urbana y comportamientos compensatorios}

\section{Compacidad y sostenibilidad: argumentos y evidencia empírica}

Existen dos discursos claramente diferenciados sobre qué modelo de ciudad resulta más sostenible, el Enfoque de Ciudad Autosuficiente y el Enfoque de Ciudad Compacta (Frey, 1999; Holden, 2004; Holden \& Norland, 2005; Jabareen, 2006; Muñiz et al., 2013; Næss, 2001). El Enfoque de Ciudad Autosuficiente defiende un modelo descentralizado, basado en ciudades pequeñas edificadas utilizando una arquitectura bioclimática, donde se consuman preferentemente los recursos de su biorregión. Dicho enfoque da más importancia a las actitudes individuales que a cualquier coeficiente de forma urbana (Bettini, 1998; Doughty \& Hammond, 1997; Girardet, 1999; Haberl, 2001; Morris, 1982; Orrskog \& Hammond, 1993; Pickett et al., 2013). En contraposición con el Enfoque de Ciudad Autosuficiente, el Enfoque de Ciudad Compacta propone densificar y mezclar funciones para ahorrar energía en la vivienda y en los desplazamientos cotidianos, y confía en las nuevas tecnologías para aligerar los problemas de congestión (CEC, 1990; Ewing, 1997; Gillham, 2002; Jabareen, 2006; Newman \& Kenworthy, 1999). En un contexto regional, el Enfoque de Ciudad Compacta apuesta por reforzar el monocentrismo tradicional (Bertaud, Lefèvre \& Yuen, 2009), o bien por seguir un modelo descentralizado pero concentrado, es decir, policéntrico (Ewing, 1997; Rogers \& Gumuchdjian, 2000). Centrados en el Enfoque de Ciudad Compacta, su desarrollo en EE.uU., Canadá o Australia pretende corregir la excesiva dispersión de sus ciudades (Brueckner, 2000; Ewing et al., 2008; Newman \& Kenworthy, 1999). En Europa, dicho enfoque 
persigue cuatro objetivos: a) reivindicar el modelo urbano europeo continental frente al estadounidense; b) estimular un discurso urbanístico demasiado anclado en cuestiones relativas al lenguaje formal de la arquitectura; c) plantear el hecho urbano heredado, no como una carga, sino como una oportunidad; y d) destacar las ventajas de una elevada densidad y de la mezcla de usos del suelo en términos de eficiencia energética (CEC, 1990; Ellin, 1999).

\section{Comportamientos compensatorios}

Son numerosos los trabajos que han detectado ventajas ambientales asociadas a la densidad. Por ejemplo, Camagni, Gibelli y Rigamonti (2002), Hillman (1996), Newman y Kenworthy (1999) y Owens (1986), detectan una correlación negativa entre densidad y consumo energético en el transporte. Cervero (1991), Burchell et al. (1998), Parshall et al. (2009) y Sierra Club (1998) muestran cómo las políticas de compacidad reducen el ritmo de conversión de suelo agrícola o forestal en suelo urbanizado. Ewing y Rong (2008), Larivière y LaFrance (1999), y Markham (2009) hallan que las tipologías de vivienda propias de la ciudad compacta requieren de un menor consumo de energía para su funcionamiento. Si se utiliza como variable dependiente un indicador de impacto global como las emisiones de GEI o la Huella Ecológica, la densidad sigue ejerciendo un efecto positivo en la mayoría de los casos (Bartaud et al., 2009; Brown, Southworth \& Sarzynski, 2008; Dodman, 2009; Glaeser \& Kahn, 2010; Muñiz \& Galindo, 2005; Norman, MacLean \&Kennedy, 2006; Romero Lankao et al., 2009). Aun siendo numerosa y variada la bibliografía favorable a los efectos medioambientalmente positivos de la densidad, conviene tener presente que: a) el efecto estimado cae sustancialmente al incluir variables socioeconómicas en el modelo econométrico; b) son diversos los estudios que no encuentran ventajas ambientales significativas asociadas a la densidad (Bento, Cropper, Mobarak \& Vinha, 2005; Levinson \& Kumar, 1997; Travisi, Camagni \& Nijkamp, 2010); y c) los estudios sobre movilidad obligada no permiten capturar la posible existencia de comportamientos compensatorios. Por consiguiente, no es posible establecer a priori cuál es el impacto de la densidad sobre el consumo de energía y las emisiones de GEI (Næss, 2005).

Diversos estudios han planteado la posibilidad de que los individuos que viven en barrios densos "compensen" una baja movilidad durante los días laborables con una mayor movilidad por motivos de ocio (Dijst, Lanzendorf, Barendregt \& Smit, 2004; Holden, 2007; Holden \& Linnerud, 2011; Módenes \& López-Colàs, 2007; Næss, 2006). Algunos autores sostienen que el hecho de que los individuos que viven en barrios densos tengan una mayor movilidad asociada al ocio no se debe a una estrategia compensatoria, sino a un sesgo de autoselección. Dicho sesgo revertiría la dirección de la causalidad clásica, según la cual el contexto urbano afecta la movilidad. La autoselección implica que las decisiones de localización se toman también teniendo en cuenta las preferencias sobre movilidad (Næss, 2006).

La investigación aplicada ha estudiado el efecto de la densidad sobre la probabilidad de tenencia o uso de una segunda residencia (Dijst \& Vermeulen, 1999; Dijst et al., 2004; Keers, 1998, Módenes \& López-Colàs, 2007; Stradell \& Hall, 2015), sobre el número de salidas durante el fin de semana (Kagermeier, 1997, Schlich \& 
Axhausen, 2002), sobre el consumo de energía en movilidad y vivienda (Holden \& Norland, 2005) y sobre la Huella Ecológica de movilidad y vivienda (Hoyer \& Holden, 2003; Muñiz et al., 2013). En la mayoría de los casos señalados se detectan comportamientos compensatorios, aunque la densidad sigue ejerciendo un efecto medioambientalmente positivo.

\section{La Huella Ecológica de las ciudades}

La Huella Ecológica mide la superficie de suelo ecológicamente productivo que se requiere de forma continuada para producir los recursos y absorber los residuos de una determinada población, dondequiera que este suelo se encuentre (Rees \& Wackernagel, 1996, 1998; Wackernagel \& Rees, 1996). La Huella Ecológica puede interpretarse como el reverso de la "capacidad de carga" de un territorio. Para el caso de los humanos, la capacidad de carga de un territorio es el nivel máximo de explotación de recursos y de generación de residuos que el territorio puede tolerar de forma indefinida sin que se deterioren sus ecosistemas (Rees, 1992). El concepto de 'huella' parte de la premisa de que una sociedad, para ser sostenible, debería vivir exclusivamente a partir de recursos renovables. En este sentido, la huella mide una superficie asociada a la capacidad regenerativa de la biosfera, es decir, la superficie fotosintética -o colector solar- necesaria para reponer la energía libre o negentropía disipada por los humanos (Ress, 1992). Bajo un enfoque de huella, la economía se considera un subsistema expansivo en contradicción con los límites físicos de la biosfera (Rees, 2000). Por consiguiente, el indicador de huella está en consonancia con la Segunda Ley de la Termodinámica (Georgecu-Rogen, 1971). Tal como se afirma en Calatayud (2010) y Calatayud y Cuesta (2010) al revisar los orígenes del indicador de Huella Ecológica siguiendo el trabajo de Carpintero y Naredo (2004), dicha huella recoge y sintetiza de forma operativa conceptos como "apropiación primaria neta del planeta” (Pfaundler, 1902), "energía fantasma” (Cottrell, 1950), "comercio de acres" (Borgstrom, 1965), "energía encarnada" (Odum, 1989), "capacidad de carga del planeta" (Catton, 1980; Vitousek et al., 1986) y "capital natural" (Costanza \& Daly, 1990). Aquello que distingue a la Huella Ecológica de otros indicadores de insostenibilidad es que los diferentes impactos se agregan con criterios ecológicos, asignándole a cada uno un espacio ecológico exclusivo (Wackernagel \& Yount, 1998).

Si bien es cierto que el cálculo de huellas plantea algunos problemas relacionados con el hecho de que un mismo espacio pueda estar suministrando diferentes funciones (Moffatt, 2000; Rapport, 2000), la literatura sobre huellas es extremadamente dinámica y periódicamente se llevan a cabo correcciones y mejoras (Monfreda, Wackernagel \& Deumling, 2004). La Huella Ecológica se obtiene a partir de la agregación de dos superficies conceptualmente diferentes: a) la superficie real que ocupan los recursos naturales utilizados; y b) la huella teórica de carbono correspondiente a la superficie de bosques necesaria para absorber los GHG (greenhouse gases) emitidos en la producción, transporte y consumo de bienes y servicios (Browne, O’Reagan \& Moles, 2009). Aplicada en un ámbito urbano, la Huella Ecológica mide el área total de ecosistemas requeridos de forma continua para suministrar las demandas materiales y absorber los residuos de la población residente 
(Rees \& Wackernagel, 1996). En la mayoría de los estudios, este cálculo arroja como resultado una superficie que puede multiplicar por 200 el espacio urbanizado. Dicha desproporción ha inspirado la metáfora de la ciudad como parásito de un amplio y disperso territorio (Odum, 1971; Vitousek et al., 1986; Wackernagel \& Yount, 1998).

Si los individuos vivieran consumiendo exclusivamente los productos y servicios del capital natural de la bioregión donde viven, entonces el planeta sería sostenible por definición. El problema es que la globalización y el comercio han creado las condiciones para que ciudades, regiones y países crezcan muy por encima de lo que les permitiría una explotación sostenible de los recursos locales (Rees, 1992; Rees \& Wackernagel, 1996). Desde una perspectiva global, la Huella Ecológica total puede reducirse consumiendo exclusivamente los recursos de la región, preservando el capital natural local y aumentado la productividad de los ecosistemas locales. El problema es que este camino conllevaría reducciones drásticas en los niveles de consumo y/o un fuerte ajuste poblacional en las regiones urbanas con mayor déficit ecológico. Otra posible estrategia consistiría en la adopción de medidas que permitieran reducir la huella per cápita a escala de individuo/ciudad/región/país, convergiendo hacia unos niveles compatibles con el capital natural existente a escala planetaria. En este contexto las políticas de compacidad podrían resultar exitosas, ya que en diversos estudios se constata la existencia de una correlación negativa entre densidad y huella per cápita (Moles, Foley, Morrissey \& O’Regan, 2008; Moos, Whitfield, Johnson \& Andrey, 2006; Muñiz \& Galindo, 2005; Muñiz et al., 2013; O’Reagan, Morrissey, Foley \& Moles, 2009; Rees \& Wackernagel, 1996; Ryu, 2005; Walker, 2013), si bien es cierto que algunos han obtenido evidencia empírica que apunta en la dirección contraria (Australian Conservation Foundation, 2007; Marique, Dujardin, Teller \& Reiter, 2013).

\section{El Área Metropolitana de Concepción (AMC) como ámbito de estudio}

El Área Metropolitana de Concepción (Chile), también llamada Gran Concepción, se encuentra en la parte centro-sur de Chile, en la costa del Pacífico. El río Biobío y los cordones de la costa han condicionado en gran medida su desarrollo urbanístico (Romero \& Vidal, 2010; Salinas, 2010). El AMC está conformado por once comunas que en conjunto ocupan $2.831 \mathrm{Km}^{2}$. Con cerca de 900.000 habitantes, se trata de la segunda área metropolitana chilena en importancia después de Santiago (Rojas, Muñiz \& Pino, 2009). Contiene un núcleo destacado, Concepción, así como una serie de subcentros, entre los cuales destacan Talcahuano y Coronel (Gysling \& Hoffman, 2010; Rojas, Muñiz \& García-López, 2009). El Área Metropolitana de Concepción actualmente se rige por el Plan Regulador Metropolitano de Concepción, vigente desde el año 2003, el cual se encuentra en proceso de actualización (http://www.prmconcepcion.cl). El crecimiento reciente especula sobre los límites propuestos en el Plan Regulador, urbanizando suelos con un elevado valor ecológico (Rojas, Pino, Basnou \& Vivanco, 2013). En Concepción abunda la ocupación alegal de terrenos, comúnmente llamados "tomas de terreno", donde la calidad de los materiales de construcción es baja. Además, tales sitios suelen estar ubicados 
en lugares con un elevado riesgo de desplazamientos de tierras debido a fenómenos sísmicos. Si bien en el Amc la baja densidad es la norma (Rojas, Salado, Pino \& Martori, 2011), en las áreas más céntricas existen edificios de entre 8 y 25 plantas y tejidos residenciales relativamente densos. De acuerdo con datos de manzanas censales del año 2002, la densidad bruta del AMc es 37,98/ha (3.798 hab./ $\mathrm{Km}^{2}$ ). Las densidades más altas se ubican en los centros consolidados de Concepción, Talcahuano y Hualpén. En cambio, las densidades más bajas se encuentran generalmente en suelos periféricos, zonas cercanas a carreteras, localidades costeras y, en general, áreas más distantes del centro principal de Concepción así como del resto de centros urbanos que estructuran el territorio metropolitano (Rojas, Muñiz \& Pino, 2009; Serpell, Kort \& Vera, 2013).

\section{Datos y metodología}

\section{El cuestionario y otras fuentes de datos}

La selección de barrios donde llevar a cabo las encuestas pretende capturar la variedad de situaciones en cuanto a densidad, centralidad y nivel de ingresos. Bajo dicho criterio, se encuestó a población residente en diecinueve barrios ${ }^{1}$ pertenecientes a ocho comunas (Concepción, Hualpén, Talcahuano, San Pedro de la Paz, Chiguayante, Coronel, Penco y Tomé). Se descartaron las comunas más rurales, Hualqui y Santa Juana, por estar pobremente integradas desde un punto de vista funcional. Tampoco se seleccionó Lota, debido a su semejanza con Coronel (figura 1). Los barrios estudiados presentan una densidad neta media de 149 hab./ha, con la menor cifra en el barrio Andalué - de alta plusvalía e ingresos altos-, en San Pedro de la Paz, con 15,75 hab./ha. En contraste, la densidad más alta se eleva a 426 hab./ha en el barrio de ingresos bajos Cerro Centinela, en Talcahuano.

Los datos sobre consumos energéticos en movilidad y vivienda, características socioeconómicas y actitudes ante la sostenibilidad provienen de la explotación de 475 encuestas sobre los patrones de consumo de los hogares (25 encuestas por barrio). Esto supuso recoger información sobre 1.662 individuos (en promedio cada hogar está compuesto por 3,5 individuos). La información contenida en los cuestionarios se agrupa en cuatro categorías: a) perfil familiar (número de miembros del hogar, renta per cápita, tipología hogar); b) actitudes respecto a la sostenibilidad (valoración ambiental del lugar de residencia y peso que tuvo en su elección); c) vivienda (superficie de la vivienda, tipología de la vivienda, consumo de energía en gas y electricidad; d) movilidad (número de desplazamientos, modo de transporte y distancia recorrida). Por último, los datos de densidad se calculan con el Censo de Población y Vivienda 2002, y el área de la sección censal mediante un Sistema de Información Geográfica (sIG). La distancia recorrida se aproxima considerando la distancia en línea recta entre origen y destino.

Se comenzó con una lista de veinte barrios, pero uno de ellos se descartó debido a la baja calidad de la información obtenida. 
FIgURA I | Mapa del Área Metropolitana de Concepción

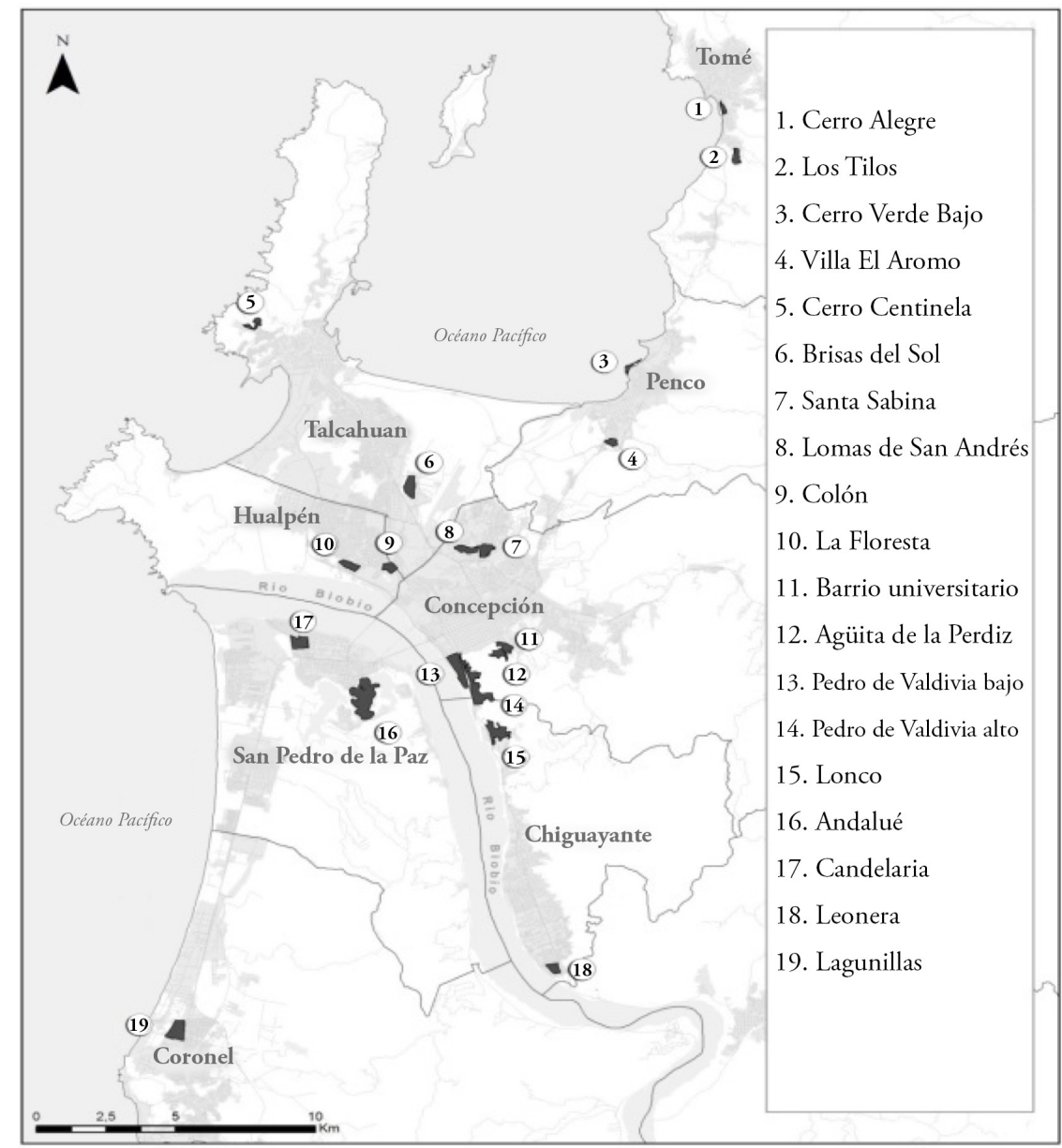

FUENTE LÍMITES ADMINISTRATIVOS INE (2002), SUPERFICIES URBANAS (ROJAS ET AL., 20I3) Y BARRIOS (ELABORACIÓN PROPIA)

\section{La medida de impacto ambiental global: la Huella Ecológica correspondiente} al Energy Land

El indicador de impacto ambiental a escala global utilizado en esta investigación es la Huella Ecológica correspondiente al Energy Land, es decir, la superficie teórica de bosques necesaria para absorber los GHG liberados a la atmósfera. Esto supone dejar fuera del cálculo de huella la superficie ocupada por las infraestructuras de transporte, debido a que no puede cargarse exclusivamente a la movilidad individual. El Energy Land, uno de los componentes de la Huella Ecológica, es equivalente a la Huella de Carbono (Pon \& Pon, 2009; Wiedmann \& Minx, 2007), una vez que se convierten en hectáreas las toneladas de $\mathrm{CO}_{2}$ utilizando el factor de conversión $5.2 \mathrm{Tn} \mathrm{CO}_{2} / \mathrm{ha}$ propuesto por el Grupo Intergubernamental de Expertos sobre el Cambio Climático 
(IPCC) (véase tabla 1). Además, la inclusión de la superficie urbanizada daría pie a posibles problemas de endogeneidad que podrían sesgar el valor de los parámetros.

TABla I | Población y densidad en los barrios seleccionados

\begin{tabular}{|c|c|c|c|c|c|}
\hline & $\begin{array}{c}\text { POBLACIÓN } \\
\text { TOTAL }\end{array}$ & $\begin{array}{c}\text { DENSIDAD } \\
\text { PROMEDIO DE } \\
\text { LA MANZANA } \\
\text { (HAB./HA) }\end{array}$ & $\begin{array}{c}\text { DENSIDAD } \\
\text { PROMEDIO DE } \\
\text { LA MANZANA } \\
\left(\text { HAB. } / \text { KM }^{2}\right)\end{array}$ & $\begin{array}{c}\text { DENSIDAD } \\
\text { DEL BARRIO } \\
(\text { HAB./HA })\end{array}$ & $\begin{array}{c}\text { DENSIDAD } \\
\text { DEL BARRIO } \\
\left(\text { HAB./KM }{ }^{2}\right)\end{array}$ \\
\hline Agüita de la Perdiz & 2.189 & 1241,41 & 11285,53 & 149,93 & 14992,60 \\
\hline Pedro de Valdivia alto & 3.538 & 56,59 & 5659,24 & 55,95 & 5595,00 \\
\hline Pedro de Valdivia bajo & 6.734 & 234,64 & 23464,14 & 154,54 & 15453,52 \\
\hline Barrio universitario & 1.432 & 107,56 & 10755,73 & 96,40 & 9639,57 \\
\hline Leonera & 2.771 & 286,72 & 28671,58 & 195,17 & 19516,87 \\
\hline Candelaria & 5.568 & 195,82 & 19582,44 & 191,63 & 19162,91 \\
\hline Villa El Aromo & 754 & 22,53 & 2253,14 & 71,80 & 7179,80 \\
\hline Cerro Verde Bajo & 1.653 & 216,73 & 21672,63 & 263,05 & 26304,62 \\
\hline Los Tilos & 965 & 143,25 & 14324,58 & 71,61 & 7160,54 \\
\hline Cerro Alegre & 910 & 100,04 & 10003,84 & 148,96 & 14896,27 \\
\hline Cerro Centinela & 4.943 & 460,60 & 46059,51 & 426,26 & 42626,38 \\
\hline La Floresta & 2.080 & 139,24 & 13924,11 & 121,38 & 12138,35 \\
\hline Colón & 2.404 & 190,35 & 19034,68 & 152,68 & 0,16 \\
\hline Lagunillas & 8.128 & 353,35 & 35335,09 & 200,09 & 20008,90 \\
\hline Lonco & 2.904 & 72,98 & 7297,90 & 75,83 & 7583,05 \\
\hline Lomas San Andrés & 2.356 & 138,15 & 13815,48 & 123,19 & 12319,05 \\
\hline Santa Sabina & 3.737 & 220,12 & 22012,25 & 171,02 & 17102,48 \\
\hline Andalué & 1.607 & 35,71 & 3570,51 & 15,75 & 1574,80 \\
\hline
\end{tabular}

FUENTE ROJAS, MUÑIZ Y PINO (2009)

Con la información sobre consumo de energía en vivienda y movilidad, se calcula la Huella Ecológica correspondiente al Energy Land de cada unidad familiar dividida por el número de miembros que la componen (Huella Ecológica total per cápita). Este valor se obtiene al sumar cuatro subhuellas:

- Huella Ecológica de los consumos energéticos residenciales

- Huella Ecológica de la movilidad obligada

- Huella Ecológica de la movilidad de fin de semana

- Huella Ecológica de la movilidad por vacaciones

La Huella Ecológica de la movilidad y de los consumos energéticos residenciales se ha estimado utilizando los factores de conversión que aparecen en la tabla 2. 
TABLA 2 $\mid$ Tabla de factores de conversión utilizados y otra información requerida para el cálculo de la Huella Ecológica

\begin{tabular}{|l|r|l|}
\hline Equivalencia TnCO2/ha & 5,2 & (IPCC) \\
\hline Electricidad (gCO2/KWh) & 248,0 & (Oficina catalana canvi climàtic, 2013 \\
\hline Gas (TnCO2/Tj) & 51,3 & (Anexo I de Decisión 2007/589/CE) \\
\hline Carbón $(\mathrm{TnCO} 2 / \mathrm{Tj})$ & 94,5 & (Anexo I de Decisión 2007/589/CE) \\
\hline Parafina $(\mathrm{TnCO} 2 / \mathrm{Tj})$ & 73,3 & (Anexo I de Decisión 2007/589/CE) \\
\hline Leña (TnCO2/Tj) & 0,0 & (Anexo I de Decisión 2007/589/CE) \\
\hline Días laborables & 225,0 & \\
\hline Hax 1000 pasajeros/Km coche & 0,08 & Chambers, Simmons \& Wackernagel $(2000)$ \\
\hline Hax 1000 pasajeros/Km bus y metro & 0,03 & Chambers, Simmons \& Wackernagel $(2000)$ \\
\hline Ha x 1000 pasajeros/Km avión & 0,09 & Chambers, Simmons \& Wackernagel $(2000)$ \\
\hline
\end{tabular}

FUENTE ELABORACIÓN PROPIA

\section{El modelo general}

A continuación se estima un modelo de regresión múltiple por Mínimos Cuadrados Ordinarios (MCO), tomando como variable dependiente el logaritmo de la Huella Ecológica de los consumos energéticos residenciales y de la movilidad per cápita. Las variables explicativas se agrupan en tres categorías: a) Variables socioeconómicas (número de miembros del hogar, renta per cápita, tipología hogar); b) Actitudes ante la sostenibilidad controles endogeneidad (valoración ambiental del lugar de residencia y peso que tuvo en su elección); y c) Forma y estructura urbana (Dummy=1 en barrios densos; distancia al СвD y distancia al СвD al cuadrado).

Se espera que el parámetro estimado de la variable número de individuos que componen el hogar presente un signo negativo, capturando la existencia de economías de escala en la provisión de servicios en el hogar. El signo esperado del parámetro correspondiente a la variable renta familiar es positivo. Se espera que el grupo de solteros tenga un impacto significativamente superior al resto de tipologías de hogar (Hoyer \& Holden, 2003; Muñiz et al., 2013). En cuanto a las actitudes ante la sostenibilidad, se preguntó a los encuestados hasta qué punto en la elección del lugar de residencia se tomó en cuenta la posibilidad de tener un bajo impacto ambiental o bien el tipo de movilidad que dicha localización conlleva. Al incluir esta información en el modelo se controlan los problemas de endogeneidad asociados a la autoselección residencial (Næss, 2006; Holden \& Linnerud, 2011).

Una vez controladas las características socioeconómicas y la posible autoselección residencial, las variables de estructura urbana deberían capturar su impacto neto sobre la Huella Ecológica per cápita. Así, por ejemplo, si el coeficiente de la densidad fuera negativo, ello implicaría que, en caso de existir comportamientos compensatorios, no serían suficientemente importantes como para anular los beneficios ambientales de la densidad en términos de huella. Si, por el contrario, el signo resulta positivo, implicaría que los comportamientos compensatorios son suficientemente importantes como para que la densidad ejerza un impacto de signo positivo (más densidad, más huella), negando con ello los beneficios ambientales asociados a la densidad. Tal hipótesis puede corroborarse examinando el signo de los parámetros de estructura urbana en el modelo correspondiente a la movilidad por ocio. 
En los modelos estimados se incluyen simultáneamente la densidad y la distancia al centro principal. Según el Modelo de Ciudad Monocéntrica (Alonso, 1964; Anas, Arnott \& Small, 1998), la densidad depende negativamente de la distancia al CBD, por lo que deberían estimarse en modelos diferentes. Sin embargo, se estimaron dichos modelos y el valor de los parámetros permaneció estable. De hecho, la correlación entre densidad y distancia al centro es inferior a 0,35 , lo cual aconseja que se estimen en un mismo modelo. También se incluyó como regresor la distancia al centro al cuadrado, con el objeto de capturar la posible existencia de máximos o mínimos locales. En caso de existir beneficios asociados a la centralidad, el signo del parámetro de la variable distancia al СвD debiera ser positivo.

\section{Resultados}

La tabla 3 presenta los valores promedio de la información obtenida a partir de 475 encuestas.

TABLA $3 \quad$ Resumen de información obtenida a partir del cuestionario

\begin{tabular}{|c|c|}
\hline & VALORES PROMEDIO \\
\hline Número de personas por hogar & 3,84 \\
\hline Superficie $\left(\mathrm{m}^{2}\right)$ vivienda & 106 \\
\hline Año de construcción de la vivienda & 1987 \\
\hline $\begin{array}{l}\text { Principales materiales utilizados en la construcción de las } \\
\text { viviendas }\end{array}$ & $\begin{array}{l}\text { Hormigón }(28 \%) \text {, mixto }(33 \%) \text {, } \\
\text { madera }(24 \%)\end{array}$ \\
\hline Renta del hogar & $\$ 561.614$ \\
\hline Tipo de gas & Bombona 15 kilos $(54 \%)$ \\
\hline Otras fuentes de energía & Leña $(83 \%)$ \\
\hline Dispone de una segunda residencia & $15 \%$ \\
\hline Se desplazan los fines de semana & $41 \%$ \\
\hline $\begin{array}{l}\text { Interés declarado sobre el tipo de movilidad que quisieran } \\
\text { realizar y hasta qué punto se tuvo en cuenta al escoger el lugar } \\
\text { de residencia (o a 10) }\end{array}$ & 4,27 \\
\hline Interés medioambiente (o a 10) & 4,27 \\
\hline Tipología del edificio & Vivienda unifamiliar (93\%) \\
\hline No automóviles del hogar & 0,78 \\
\hline Distancia promedio al lugar de trabajo & 13,65 \\
\hline Porcentaje de uso de automóvil para ir al lugar de trabajo & 0,21 \\
\hline
\end{tabular}

FUENTE ELABORACIÓN PROPIA

\section{Los valores de Huella Ecológica}

Los cálculos llevados a cabo arrojan un valor promedio de Huella Ecológica en movilidad individual y consumos energéticos residenciales de 0,106 ha/hab. (0,55 toneladas de GEI/hab.) (tabla 4). Dicho valor está sensiblemente por debajo 
de la huella estimada en otros trabajos que han adoptado una metodología similar. ${ }^{2}$ El tamaño de la huella de los consumos energéticos residenciales se debe principalmente al consumo de energía eléctrica. En el caso de la movilidad individual, la movilidad obligada (commuting y estudios) es la que presenta una mayor huella. La Huella Ecológica per cápita de los barrios con una elevada renta per cápita es aproximadamente el doble de la correspondiente a los barrios con una baja renta per cápita. Dada la población censada en Concepción (algo más de 874.000 personas según el Censo 2002), la huella total asociada a la movilidad y a los consumos energéticos residenciales sería de 92.700 hectáreas. Tal es la superficie de bosques necesaria para absorber las 482.000 toneladas de GHG que se calcula emite la población de Concepción debido a la movilidad individual y al consumo de energía en las viviendas. Esta superficie representa aproximadamente un tercio de la superficie total del AMC (283.100 ha).

TABla 4 Resultados del cálculo de la Huella Ecológica de la movilidad individual y de los consumos energéticos residenciales

\begin{tabular}{|l|c|}
\hline \multicolumn{1}{|c|}{$\begin{array}{c}\text { HUELLA ECOLÓGICA } \\
\text { DE CONSUMOS RESIDENCIALES }\end{array}$} & (HA/HAB) \\
\hline Gas & 0,007 \\
\hline Electricidad & 0,042 \\
\hline Otras fuentes & 0,004 \\
\hline Huella residencial total & 0,055 \\
\hline Huella Ecológica movilidad individual & \\
\hline Movilidad obligada (trabajo y estudios) & 0,042 \\
\hline Movilidad vacaciones & 0,004 \\
\hline Movilidad fin de semana & 0,004 \\
\hline Huella movilidad total & 0,051 \\
\hline Huella Ecológica total (movilidad y vivienda) & 0,106 \\
\hline
\end{tabular}

FUENTE ELABORACIÓN PROPIA

\section{Las regresiones}

Se han estimado cuatro modelos, cuyos resultados se muestran en la tabla 5. En la primera columna aparecen los resultados correspondientes al modelo estimado, utilizando como variable dependiente la huella ecológica de la movilidad obligada; en la segunda, la huella de la movilidad por ocio (fin de semana y vacaciones); en la tercera, la huella total de la movilidad (suma de las dos anteriores); en la cuarta, la huella de los consumos energéticos de la vivienda; y en la quinta, la huella total de la movilidad y de la vivienda. Los valores de huella se toman en términos per cápita y en logaritmos, lo cual permite interpretar directamente como elasticidades los parámetros de las variables explicativas expresadas también en logaritmos. Se llevaron a cabo estimaciones sin aplicar logaritmos, y los resultados fueron muy

2 El valor promedio es aproximadamente un tercio del obtenido para el caso de la Región Metropolitana de Barcelona (Muñiz et al., 2013). 
semejantes en cuanto al signo y a la significatividad de los parámetros. Las variables explicativas comunes para los cinco modelos estimados son: el número de miembros de cada hogar, la renta per cápita, una dummy que toma valor uno en caso de ser un barrio denso (una densidad superior a la media $149 \mathrm{ha} / \mathrm{hab}.),{ }^{3}$ la distancia al CBD, la distancia al СвD al cuadrado. En las primeras pruebas se añadieron como variables explicativas el número de automóviles y la superficie de la vivienda, pero estaban demasiado correlacionadas con la renta per cápita, por lo que fueron finalmente descartadas. Por último, ninguna tipología familiar resultó significativa.

Los parámetros estimados para las variables socioeconómicas presentan el signo esperado. El número de habitantes por vivienda ejerce un impacto negativo sobre la huella per cápita, lo que confirma la existencia de economías de escala en el consumo de energía de la vivienda y también en la movilidad, especialmente la de ocio y vacaciones. Siendo el principal factor que explica la variabilidad observada en cada una de las subhuellas, la renta per cápita ejerce un impacto de signo positivo (al aumentar la renta la huella crece) y significativo en los cinco modelos. Los controles de autoselección presentan por lo general el signo esperado, aunque resultan poco significativos.

TABLA 5 | Estimaciones modelos econométricos estimados mediante Mínimos Cuadrados Ordinarios (MCO)

\begin{tabular}{|c|c|c|c|c|c|}
\hline & $\begin{array}{c}\text { I } \\
\text { MOVILIDAD } \\
\text { OBLIGADA } \\
\text { (LN HUELLA PC) }\end{array}$ & $\mid \begin{array}{c}2 \\
\text { MOVILIDAD } \\
\text { OCIO } \\
\text { (LN HUELLA PC) }\end{array}$ & $\begin{array}{c}3 \\
\text { MOVILIDAD } \\
\text { TOTAL } \\
\text { (LN HUELLA PC) }\end{array}$ & (LN HUELLA PC) & $\begin{array}{c}5 \\
\text { TOTAL } \\
\text { VIVIENDA Y } \\
\text { MOVILIDAD } \\
\text { (LN HUELLA PC) }\end{array}$ \\
\hline $\begin{array}{l}\text { Habitantes por } \\
\text { vivienda }\end{array}$ & $\begin{array}{l}-0,075^{* *} \\
(1,8)\end{array}$ & $\begin{array}{l}-0,32^{* * * *} \\
(8,5)\end{array}$ & $\begin{array}{l}-0,08^{* *} \\
(1,9)\end{array}$ & $\begin{array}{l}-0,46^{* * * *} \\
(23,7)\end{array}$ & $\begin{array}{l}-0,15^{\text {**** }} \\
(6,9)\end{array}$ \\
\hline Ln renta familiar & $\begin{array}{l}0,33^{\text {**** }} \\
(4,5)\end{array}$ & $\begin{array}{l}0,49^{* * * *} \\
(7,2)\end{array}$ & $\begin{array}{l}0,48^{* * * *} \\
(7,2)\end{array}$ & $\begin{array}{l}0,176^{* * * *} \\
(6,6)\end{array}$ & $\begin{array}{l}0,29^{* * * *} \\
(8,8)\end{array}$ \\
\hline Densidad alta $(=1)$ & $\begin{array}{l}-0,142 \\
(1,1)\end{array}$ & $\begin{array}{l}-0,015 \\
(0,1)\end{array}$ & $\begin{array}{l}0,06 \\
(0,5) \\
\end{array}$ & $\begin{array}{l}-0,02 \\
(0,5)\end{array}$ & $\begin{array}{l}-0,03 \\
(0,5)\end{array}$ \\
\hline Ln dist СвD & $\begin{array}{l}1,26^{* * * *} \\
(4,0)\end{array}$ & $\begin{array}{l}0,66^{* * *} \\
(2,2)\end{array}$ & $\begin{array}{l}0,97^{* * * *} \\
(3,6)\end{array}$ & $\begin{array}{c}0,002 \\
(0,01) \\
\end{array}$ & $\begin{array}{l}0,207^{*} \\
(1,5)\end{array}$ \\
\hline Ln dist $\mathrm{CBD}^{2}$ & $\begin{array}{l}-0,22^{* * *} \\
(2,6)\end{array}$ & $\begin{array}{l}-0,19^{* * *} \\
(2,34)\end{array}$ & $\begin{array}{l}-0,17^{* * *} \\
(2,2)\end{array}$ & $\begin{array}{l}-0,02 \\
(0,7) \\
\end{array}$ & $\begin{array}{l}-0,04 \\
(1,0)\end{array}$ \\
\hline $\begin{array}{l}\text { Controles } \\
\text { autoselección }\end{array}$ & sí & sí & sí & sí & sí \\
\hline Constante & $\begin{array}{l}-2,7^{* * * *} \\
(2,9)\end{array}$ & $\begin{array}{l}-4,09^{* * * *} \\
(4,0)\end{array}$ & $\begin{array}{l}-4,5^{* * * *} \\
(5,0)\end{array}$ & $\begin{array}{l}4,5^{\text {**** }} \\
(11,8)\end{array}$ & $\begin{array}{l}0,63^{*} \\
(1,5)\end{array}$ \\
\hline $\mathrm{R}^{2}$ ajustado & 0,19 & 0,31 & 0,26 & 0,65 & 0,35 \\
\hline No Observaciones & 475 & 475 & 475 & 475 & 475 \\
\hline
\end{tabular}

3 Los barrios que presentan una densidad superior a la media son: Cerro Verde bajo, Cerro Centinela, Santa Sabina, Colón, Agüita La Perdiz, Pedro Valdivia bajo, Candelaria y Lagunillas. 
En cuanto a las variables asociadas a la forma y estructura urbana, es especialmente destacable el nulo efecto que parece ejercer la densidad sobre las diferentes subhuellas. La baja significatividad de la dummy para el barrio denso en el modelo más agregado podría indicar la existencia de comportamientos compensatorios. Sin embargo, el parámetro correspondiente a dicha variable no presenta un signo positivo en la estimación correspondiente a la huella de la movilidad por ocio y vacaciones, por lo que tal hipótesis debe rechazarse. Por último, la distancia al CBD ejerce un efecto de signo positivo sobre la huella de la movilidad -tanto la obligada como la de ocio y vacaciones-, por lo que tampoco se detectan comportamientos compensatorios asociados a un "exceso de centralidad" y sí, en cambio, un castigo en términos de huella para las localizaciones más periféricas. Se detecta además un efecto cuadrático significativo, por lo que, aunque la huella crece con la distancia al СBD, deja de hacerlo a partir de algún punto. No se ha hallado evidencia empírica favorable a los efectos medioambientalmente positivos asociados a la densidad, lo cual cuestionaría la efectividad de las políticas de densificación.

\section{Resumen y conclusiones}

Los valores de huella estimados son significativamente inferiores a los obtenidos en otros trabajos, lo cual puede explicarse a partir de factores como el clima o la diferencia en el nivel de ingresos. Los resultados del ejercicio econométrico indican que las variables de forma y estructura urbana ejercen un bajo impacto sobre el valor de la huella y que la distancia al $C B D$ incide en mayor medida sobre el valor de la huella que la densidad. La distancia al $C B D$ es muy significativa en los modelos estimados para la movilidad. Con anterioridad se ha comprobado que en los barrios de bajos ingresos de Concepción, la localización de los hogares, el trabajo o los estudios tienen un valor gravitante en la definición del espacio de actividades diarias. En cambio, en el caso de los hogares con un elevado nivel de ingreso, la distancia no ha supuesto un problema debido al uso preponderante del automóvil (Lima, Carrasco \& Rojas, 2014). La dummy para barrio denso no resulta en ningún caso significativa. Por consiguiente, es la localización, más que la densidad del asentamiento, lo que determina el valor de la huella. Desde esta perspectiva puede argumentarse que una política que tenga como objetivo frenar la suburbanización reduciría la emisión de GEI.

Una posible explicación para la baja significatividad de la dummy para barrios densos es la existencia de comportamientos compensatorios. Si tales comportamientos se dieran, el parámetro de la variable dummy para barrio denso en el modelo para la movilidad no obligada debería presentar un signo positivo. Sin embargo, el parámetro estimado no resulta significativo, por lo que tal hipótesis debe rechazarse. Los resultados obtenidos en esta investigación sugieren que las posibles situaciones favorables a la existencia de comportamientos compensatorios (tamańo de la ciudad, renta per cápita, etcétera) pueden ser bastante limitadas.

En el caso de Concepción, la variabilidad de las huellas estimadas responde más a diferencias de renta que a diferencias de densidad. Aunque la variabilidad en los niveles de huella por barrios es limitada, pueden detectarse algunos patrones, como, 
por ejemplo, que lo barrios con una elevada renta per cápita presentan una huella que es aproximadamente el doble que la de los barrios con una baja renta per cápita.

El trabajo llevado a cabo presenta algunas limitaciones que pretendemos corregir en investigaciones futuras. La primera es que las distancias estimadas para la movilidad son distancias aéreas (distancia más corta en línea recta entre dos puntos), es decir, no son distancias en ruta, por lo que se infravalora la distancia real y con ello también la huella de la movilidad. La segunda es que no se ha contabilizado la energía gris del edificio, es decir, el $\mathrm{CO}_{2}$ incorporado en los materiales dividido por el periodo de vida promedio de las viviendas, o bien el $\mathrm{CO}_{2}$ asociado a las nuevas construcciones. La razón de no incorporar esta fuente de huella es que no disponíamos de ningún trabajo de referencia para Chile sobre la energía ni $\mathrm{CO}_{2}$ incorporado en los materiales utilizados para la edificación de viviendas. Estas dos limitaciones suponen que la huella estimada subestima sistemáticamente el valor de la huella real.

\section{Agradecimientos}

A los profesores Hugo Capellá y Claudia García-Lima por su generosa ayuda durante todo el proyecto; a Adriana Díaz y a Juan Andrés, por su amistad y colaboración; y al Departamento de Geografía de la Universidad de Concepción (Chile), por habernos abierto sus puertas de par en par.

Los autores agradecen el apoyo prestado por la Comisión Nacional de Investigación Científica y Tecnológica (CONICYT), Fondo de Financiamiento de Centros de Investigación en Áreas Prioritarias (FONDAP), Proyecto 15110020.

\section{Referencias bibliográficas}

Alonso, W. (1964). Location and land use. Toward a general theory of land rent. Cambridge, MA: Harvard University Press.

Anas, A., Arnott, R. \& Small, K. A. (1998). Urban spatial structure. Journal of Economic Literature, 36 (3), 1426-1464. En http://www.socsci.uci.edu/-ksmall/JEL\%20Paper. pdf

Angel, S., Sheppard, S. \& Civco, D. (2005). The dynamics of global urban expansion. Washington D.c.: World Bank, Transport and Urban Development Department. En http://bit. ly/25wBiLS

Australian Conservation Foundation. (2007). Consuming Australia: Main Findings. Sydney: Autor. En https://www.acfonline.org.au/sites/default/files/resource/res_Atlas_Main_ Findings.pdf

Bento, A. M., Cropper, M. L., Mobarak, A. M. \& Vinha, K. (2005). The effects of urban spatial structure on travel demand in the United States. Review of Economics and Statistics, 87 (3), 466-478. doi: 10.1162/0034653054638292 
Bertaud, A., Lefèvre, B., \& Yuen, B. (2009). GHG emissions. Urban mobility and efficiency of urban morphology: A hypothesis. En Marseille Symposium Report 5th Urban research symposium on cities and climate change: responding to an urgent agenda. Marseille, France, 28-30 June, 2009 (pp. 28-30). En http://www.gcp-urcm.org/Resources/ R201108110025

Bettini, V. (1998). Elementos de ecología urbana. Ed. M. P. Lorca. Madrid: Trotta.

Borgstrom, G. (1965). The hungry planet: The modern world at the edge of famine. New York: Collier Books.

Brown, M. A., Southworth, F. \& Sarzynski, A. (2008). Shrinking the carbon footprint of metropolitan America. Washington, D.c.: Brookings Institution.

Browne, D., O’Regan, B. \& Moles, R. (2009). Assessment of total urban metabolism and metabolic inefficiency in an Irish city-region. Waste Management, 29 (10), 2765-2771. En http://dx.doi.org/10.1016/j.wasman.2009.05.008

Brueckner, J. K. (2000). Urban sprawl: Diagnosis and remedies. International Regional Science Review, 23 (2), 160-171. doi: 10.1177/016001700761012710

Burchell, R. W., Shad, N. A., Listokin, D., Phillips, H., Downs, A., Seskin, S., Davis, J. S., Moore, T., \& ECONort-west. (1998). The cost of sprawl revisited. TCRP Report 39. Washington D.C.: National Academy Press. En http://onlinepubs.trb.org/onlinepubs/ tcrp/tcrp_rpt_39-a.pdf

Calatayud, D. (2010). Descarbonitzar l'urbanisme. Revista Medi Ambient; (45), 31-41. En http://www.raco.cat/index.php/MATiC/article/view/297296

Calatayud, D. \& Cuesta, A. (2010). Els savis que investiguen: història de la petjada ecològica. En T. Solanas, D. Calatayud \& E. Claret (Eds.), $34 \mathrm{~kg} \mathrm{de} \mathrm{CO} 2$ (pp. 198-223). Barcelona: Generalitat de Catalunya. Departament de Medi Ambient i Habitatge.

Camagni, R., Gibelli, M. C. \& Rigamonti, P. (2002). Urban mobility and urban form: The social and environmental costs of different patterns of urban expansion. Ecological Economics, 40 (2), 199-216. http://dx.doi.org/10.1016/S0921-8009(01)00254-3

Carpintero, Ó. \& Naredo, J. M. (2004). El metabolismo de la economía española. Flujo de energía, materiales y su incidencia ecológica. En B. Halweil \& L. Mastny (Dirs.), La situación del mundo 2004: La sociedad de consumo. Informe anual del Worldwatch Institute sobre el progreso hacia una sociedad sostenible (pp. 321-349). Barcelona: Icaria; Centro de Investigación para la Paz. En http://bit.ly/1WV5BGV

Catton, W. R. (1982). Overshoot: The ecological basis of revolutionary change. Champaign, IL: University of Illinois Press.

Cervero, R. (1991). Congestion relief: The land use alternative. Journal of Planning Association and Research, 10 (2), 119-129. doi: 10.1177/0739456X9101000209

Commission of the European Communities (CEE). (1990). Green Paper on the Urban Environment. Eur 12902, Brussels: Autor. En http://bit.ly/1TS5NTN

Costanza, R. \& Daly, H. E. (1992). Natural capital and sustainable development. Conservation biology, 6 (1), 37-46. doi: 10.1046/j.1523-1739.1992.610037.x

Cottrell, W. F. (1955). Energy and Society. New York: McGraw-Hill.

Chambers, N., Simmons, C. \& Wackernagel, M. (2000). Sharing nature's interest: ecological footprints as an indicator of sustainability. London: Routledge. 
Dijst, M. \& Vermeulen, B. (1999). The second home as a challenge for spatial policy? A study into the compensation hypothesis. En Colloquium Vervoersplanologisch Speurwerk 1999.

Dijst, M., Lanzendorf, M., Barendregt, A. \& Smit L. (2004). Second homes in Germany and the Netherlands: Ownership and travel impact explained. Tijdschrift voor Economische en Sociale Geografie, 96 (2), 139-152. doi: 10.1111/j.1467-9663.2005.00446.x

Dodman, D. (2009). Blaming cities for climate change? An analysis of urban greenhouse gas emissions inventories. Environment and Urbanization, 21 (1), 185-201. doi: $10.1177 / 0956247809103016$

Doughty, M. R. C. \& Hammond, G. P. (1997). The use of environmental footprints to evaluate the sustainability of cities. En Proceedings of the Sixth IRNES Conference: Technology, the Environment and Us. Imperial College, London (pp. 170-176).

Ellin, N. (1999). Postmodern urbanism. New York: Princeton Architectural Press.

Ewing, R. (1997). Is Los Angeles-style sprawl desirable? Journal of the American planning association, 63 (1), 107-126. doi: 10.1080/01944369708975728

Ewing, R., Bartholomew, K., Winkelman, S., Walters, J. \& Chen, D. (2008). Growing Cooler: the Evidence on Urban Development and Climate Change. Washington, D.C.: Urban Land Institute.

Ewing, R., \& Rong, F. (2008). The impact of urban form on us residential energy use. Housing Policy Debate, 19 (1), 1-30. doi: 10.1080/10511482.2008.9521624

Frey, H. (1999). Designing the city: Towards a more sustainable urban form. London/New York: Spron Press.

Georgescu-Roegen, N. (1971). The entropy law and the economic process. Cambridge, MA: Harvard University Press.

Gillham, O. (2002). The limitless city: A primer on the urban sprawl debate. Washington D.C.: Island Press.

Girardet, H. (1999). Creating sustainable cities (No. 2). White River Junction, vT: Chelsea Green Publishing.

Glaeser, E. L. \& Kahn, M. E. (2010). The greenness of cities: Carbon dioxide emissions and urban development. Journal of Urban Economics, 67 (3), 404-418. doi: 10.3386/ w14238

Gravagnuolo, B. (1998). Historia del urbanismo en Europa 1750-1960. Madrid: Akal.

Gysling, I. \& Hoffman, A. (2010). Planificación intercomunal y comunal del Área Metropolitana de Concepción. Los planes de los 80. En L. Pérez \& R. Hidalgo (Eds.), Concepción metropolitano. Evolución y desafios. Santiago, Chile: Editorial Universidad de Concepción.

Haberl, H. (2001). The energetic metabolism of societies, Part I: Accounting concepts. Journal of Industrial Ecology, 5 (1), 11-33. doi: 10.1162/108819801753358481

Hillman, M. (1996). In favor of the compact city. En M. Jenks, E. Burton \& K. Williams (Eds.), The compact city: A sustainable urban form? (pp. 36-44.). London: E \& FN Spon.

Holden, E. (2004). Ecological footprints and sustainable urban form. Journal of Housing and the Built Environment, 19 (1), 91-109.

Holden, E. (2007). Achieving sustainable mobility: Every day and leisure-time travel in the UE Aldershot: Ashgate. 
Holden, E. \& Norland, I. T. (2005). Three challenges for the compact city as a sustainable urban form: Household consumption of energy and transport in eight residential areas in the Greater Oslo region. Urban Studies, 42 (12), 2145-2166. doi: 10.1080/00420980500332064

Holden, E. \& Linnerud, K. (2011). Troublesome leisure travel: The contradictions of three sustainable transport policies. Urban Studies, 48 (14), 3087-3106

Høyer, K. G. \& Holden, E. (2003). Household consumption and ecological footprints in Norway: Does urban form matter? Journal of Consumer Policy, 26 (3), 327-349. doi: 10.1023/A:1025680422704

Jabareen, Y. R. (2006). Sustainable urban forms. Their typologies, models and concepts. Journal of Planning Education and Research, (26), 38-52. doi: 10.1177/0739456X05285119

Kagermeier, A. (1997). Settlement structure and mobility: An empirical study of the case of South Bavaria. Dortmund: Dormunder Vetrieb für Bau und Planungs literatur.

Keers, G. (1998). Meer dan een Woning. (More than one property). Amersfoort, The Netherlands: Verslag Workshop.

Lariviere, I. \& Lafrance, G. (1999). Modelling the electricity consumption of cities: Effect of urban density. Energy Economics, 21 (1), 53-66. En http://dx.doi.org/10.1016/S01409883(98)00007-3

Legg, R., Moore, J., Kissinger, M. \& Rees, W. (2013). A greenhouse gas emissions inventory and ecological footprint analysis of Metro Vancouver residents' air travel. Environment and Pollution, 2 (4), 123-134. En http://dx.doi.org/10.5539/ep.v2n4p123

Levinson, D. M. \& Kumar, A. (1997). Density and the journey to work. Growth and change, 28 (2), 147-172. En http://nexus.umn.edu/papers/Density.pdf

Lima, C., Carrasco, J. \& Rojas, C. (2014). El contexto urbano y las interacciones sociales: dualidad del espacio de actividades de sectores de ingresos altos y bajos en Concepción, Chile. EURE, 40 (121), 75-99. En http://www.scielo.cl/pdf/eure/v40n121/art04.pdf

Marique, A. F., Dujardin, S., Teller, J. \& Reiter, S. (2013). School commuting: The relationship between energy consumption and urban form. Journal of transport Geography, 26, 1-11. En http://dx.doi.org/10.1016/j.jtrangeo.2012.07.009

Markham, V. (2009). U.S. population, energy and climate change. New Canaan, Ст: Center for Environment and Population. En http://www.cepnet.org/documents/us-PopulationEnergy-Climate_Change2009.pdf

McGregor, D., Simon, D. \& Thompson, D. (Eds.). (2006) The peri-urban interface: Approaches to sustainable natural and human resource use. London: Earthscan.

Módenes, J. A. \& López-Colás, J. (2007). Second homes and compact cities in Spain: Two elements of the same system? Tijdschrift voor Economische en Sociale Geografie, 98 (3), 325-335. doi: 10.1111/j.1467-9663.2007.00400.x

Moffatt, I. (2000). Ecological footprints and sustainable development. Ecological Economics, 32 (3), 359-362. doi: 10.1016/S0921-8009(99)00154-8

Monfreda, C., Wackernagel, M. \& Deumling, D. (2004). Establishing national natural capital accounts based on detailed ecological footprint and biological capacity assessments. Land Use Policy, 21 (3), 231-246. doi: 10.1016/j.landusepol.2003.10.009

Moos, M., Whitfield, J., Johnson, L. \& Andrey, J. (2006). Does design matter? The ecological footprint as a planning tool at the local level. Journal of Urban Design, 11 (2), 195-224. doi: $10.1080 / 13574800600644381$ 
Moles, R., Foley, W., Morrissey, J. \& O’Regan, B. (2008). Practical appraisal of sustainable development-Methodologies for sustainability measurement at settlement level. Environmental Impact Assessment Review, 28 (2-3), 144-165. En http://dx.doi. org/10.1016/j.eiar.2007.06.003

Morris, D. (1982). Self-reliant cities: Energy and the transformation of urban America. San Francisco, CA: Sierra Club Books.

Muñiz, I. \& Galindo, A. (2005). Urban form and the ecological footprint of commuting. The case of Barcelona. Ecological Economics, 55 (4), 499-514. En http://dx.doi. org/10.1016/j.ecolecon.2004.12.008

Muñiz, I., Calatayud, D. \& Dobaño, R. (2013). The compensation hypothesis in Barcelona measured through the ecological footprint of mobility and housing. Landscapeand Urban Planning, 113, 113-119. En http://dx.doi.org/10.1016/j.landurbplan.2013.02.004

Næss, P. (2001). Urban planning and sustainable development. European Planning Studies, 9 (4), 503-524. doi: 10.1080/713666490

Næss, P. (2005). Residential location affects travel behaviour - but how and why? The case of Copenhagen. Progress in Planning, 63, 167-257.

Næss, P. (2006). Are short daily trips compensated by higher leisure mobility? Environment and Planning B; Planning and Design, 33, 197-220.

Newman, P. (2014). Density: The sustainability multiplier: Some myths and truths with application to Perth, Australia. Sustainability, 6 (9), 6467-6487. doi: 10.3390/ su6096467

Newman, P. W. G. \& Kenworthy, J. R. (1999). Sustainability and cities. Overcoming automobile dependence. Washington, D.c./Covelo, CA: Island Press.

Norman, J., MacLean, H. L. \& Kennedy, C. A. (2006). Comparing high and low residential density: Life-cycle analysis of energy use and greenhouse gas emissions. Journal of Urban Planning and Development, 132 (1), 10-21. doi: 10.1061/(ASCE)07339488(2006)132:1(10)

Odum, H. T. (1971). Environment, power, and society (Vol. 130). New York: Wiley-Interscience.

Odum, E. P. (1989). Ecology and our endangered life-support systems. Sunderland, MA: Sinauer Associates.

Odum, H. T. (1994). Ecological and general systems: an introduction to systems ecology. Boulder, co: University Press of Colorado.

O’Regan, B., Morrissey, J., Foley, W. \& Moles, R. (2009). The relationship between settlement population size and sustainable development measured by two sustainability metrics. Environmental Impact Assessment Review, 29 (3), 169-178. doi: 10.1016/j. eiar.2008.08.002

Orrskog, L. \& Hammond, G. P. (1993). Planering foer uthaallighet - fraan kunskap till handling. R-Byggforskningsraadet (Sweden), no., 1993, 57.

Owens, S. E. (1986). Energy, planning and urban form. London: Pion.

Pickett, S. T. A., Boone, C. G., McGrath, B. P., Cadenasso, M. L., Childers, D. L., Ogden, L. A., \& Grove, J. M. (2013). Ecological science and transformation to the sustainable city. Cities, 32 (Suppl. 1), 10-20. En http://dx.doi.org/10.1016/j.cities.2013.02.008 
Parshall, L., Gurney, K., Hammer, S. A., Mendoza, D., Zhou, Y. \& Geethakumar, S. (2010). Modeling energy consumption and $\mathrm{CO}_{2}$ emissions at the urban scale: Methodological challenges and insights from the United States. Energy Policy, 38 (9), 4765-4782. doi: 10.1016/j.enpol.2009.07.006

Peters, G. P. (2008). From production-based to consumption-based national emission inventories. Ecological Economics, 65 (1), 13-23. En http://dx.doi.org/10.1016/j. ecolecon.2007.10.014

Pfaundler, L. (1902). Die Weltwirschaft im Lichte der Physik. Deutsche Revue, (22), 171-182.

Pon, D. \& Pon, J. (2009). Evaluando el impacto climático del consumo: la huella de carbono. Ecosostenible, (50), 4-15. En http://bit.ly/1X9nQJf

Rapport, D. J. (2000). Ecological footprints and ecosystem health: Complementary approaches to a sustainable future. Ecological Economics, 32 (3), 367-370. doi: 10.1016/S09218009(99)00156-1

Rees, W. E. (1992). Eclogical footprints and appropriated carrying capacity: What urban economics leaves out. Environment and Urbanization, 4 (2), 120-130. doi: $10.1177 / 095624789200400212$

Rees, W. E. (2000). Eco-footprint analysis: Merits and brickbats. Ecological Economics, 32 (3), 371-374. doi: 10.1016/S0921-8009(99)00157-3

Rees, W. \& Wackernagel, M. (1996). Our Ecological Footprint. The New Catalyst Bioregional Series. Gabriola Island, вс, Canada: New Society Publishers.

Rees, W., \& Wackernagel, M. (2008). Urban ecological footprints: why cities cannot be sustainable and why they are a key to sustainability. In Urban Ecology (pp. 537-555). Springer us.

Rogers, R. \& Gumuchdjian, P. (2000). Ciudades para un pequeño planeta. Barcelona: Gustavo Gili.

Rojas, C., Muñiz, I. \& García-López, M. Á. (2009). Estructura urbana y policentrismo en el Área Metropolitana de Concepción. EURE, 35 (105), 47-70. En http://dx.doi. org/10.4067/S0250-716120090002000003

Rojas, C., Muñiz, I. \& Pino, J. (2009), Cartography and spatial analysis of urban sprawl. Proccedings of the 24th International Cartographic Conference, Santiago, Chile. En http://icaci.org/files/documents/ICC_proceedings/ICC2009/html/refer/5_2.pdf

Rojas, C., Salado, M. J., Pino, J. \& Martori, J. C. (2011). Medidas para la valoración de la sostenibilidad de los territorios metropolitanos: aplicación al Área Metropolitana de Concepción. Boletin de la Asociación de Geógrafos Españoles, (55), 81-99. En http://age. ieg.csic.es/boletin/55/04\%20AGE\%2055.pdf

Rojas, C., Pino, J., Basnou, C. \& Vivanco, M. (2013). Assessing land-use and cover changes in relation to geographic factors and urban planning in the Metropolitan Area of Concepción (Chile). Implications for biodiversity conservation. Applied Geography, 39, 93-103. http://dx.doi.org/10.1016/j.apgeog.2012.12.007

Romero, H. \& Vidal, C. (2010). La urbanización de las cuencas de los ríos Biobío y Andalién y sus efectos sobre los riesgos de inundación y anegamientos de la ciudad de Concepción. En L. Pérez \& R. Hidalgo (Eds.), Concepción metropolitano. Evolución y desafios. Santiago, Chile: Editorial Universidad de Concepción. 
Romero, P. (2007). Are we missing the point? Particularities of urbanization, sustainability and carbon emissions in Latin American cities. Environment and Urbanization, 19 (1), 1-17. doi: 10.1177/0956247807076915

Romero, P., López Villafranco, H., Rosas Huerta, A., Gunther, G. \& Correa Armenta, Z. (Eds.). (2005). Can cities reduce global warming? Urban development and carbon cycle in Latin America. México: Inter-American Institute for Global Change Research (IAI), Universidad Autónoma Metropolitana (UAM)-Xochimilco/International Human Dimensions Programme on Global Environmental Change (IHDP)/Global Carbon Project (GCP). En http://digital.library.unt.edu/ark:/67531/metadc11934/

Ryu, H. C. (2005). Modeling the per capita ecological footprint for Dallas County, Texas: Examining demographic, environmental value, land-use and spatial influences. Tesis doctoral. Texas, A\&M University.

Salinas, E. (2010). Procesos urbanos recientes en el Área Metropolitana de Concepción. En L. Pérez \& R. Hidalgo (Eds.), Concepción metropolitano. Evolución y desafios. Santiago, Chile: Editorial Universidad de Concepción.

Satterthwaite, D. (2008). Cities' contribution to Global Warming: Notes on the allocation of greenhouse emissions. Environment and Urbanization, 20 (2) 539-549. doi: $10.1177 / 0956247808096127$

Schlich, R. \& Axhausen, K. W. (2002). Wohnumfeld und Freizeitverkehr eine Untersuchung zur Fluchttheorie (Living environment and leisure trade, a theory of escape). Arbeitsberichte Verkehr und Raumplanung, 155, IVT, ETH, Zurich.

Serpell, A., Kort, J. \& Vera, S. (2013). Awareness, actions, drivers and barriers of sustainable construction in Chile. Technological and Economic Development of Economy, 19 (2), 272-288. doi: 10.3846/20294913.2013.798597

Sierra Club. (1998). Sprawl: The dark side of the American dream. En http://www.sierraclub. org/sprawl/report98/what.html

Strandell, A. \& Hall, C. M. (2015). Impact of the residential environment on second home use in Finland -Testing the Compensation hypothesis. Landscape and Urban Planning, 133, 12-23. En http://dx.doi.org/10.1016/j.landurbplan.2014.09.011

Travisi, C. M., Camagni, R. \& Nijkamp, P. (2010). Impacts of urban sprawl and commuting: A modelling study for Italy. Journal of Transport Geography, 18 (3), 382-392. En http:// dx.doi.org/10.1016/j.jtrangeo.2009.08.008

un-Habitat (United Nations Human Settlement Programme). (2012). Cities and climate change. Global Report on Human Settlements 2011. London/Washington, D.C.: Autor.

United Nations Population Fund (UNFPA). (2007). Estado de la población mundial, 2007. Liberar el potencial de crecimiento urbano. [Online]. En https://www.unfpa.org/sites/ default/files/pub-pdf/swp2007_spa.pdf

Vitousek, P. M., Ehrlich, P. R., Ehrlich, A. H. \& Matson, P. A. (1986) Human appropriation of the products of the photosynthesis. BioScience, 36 (6), 368-373. doi:10.2307/1310258

Wachernagel, M. \& Rees, W. (1996). Our ecological footprint. Gabriola Island, BC, Canada: New Society Publishers.

Wackernagel, M. \& Yount, J. D. (1998). The ecological footprint: An indicator of progress towards regional sustainability. Environmental Monitoring and Assessment, (51), 511529. 
Walker, L. (2013). Ecovillage at Ithaca: Pioneering a sustainable culture. Gabriola Island, BC, Canada: New Society Publishers.

Walraven, A. (2009). The impact of cities in terms of climate change. Nairobi, Kenya: United Nations Environment Programme.

Wiedmann, T. \& Minx, J. (2007, junio). A definition of "carbon footprint". ISA UK Research report 07-01. En http://citeseerx.ist.psu.edu/viewdoc/download?doi=10.1.1.467.682 $1 \&$ rep=rep1\&type=pdf [Publicado en C. C. Pertsova, Ecological Economics Research Trends (Chapter 1, pp. 1-11). Hauppauge, NY: Nova Science Publishers, 2008]. 